\title{
Towards the Complexity of Recognizing Pseudo-intents
}

\author{
Barış Sertkaya \\ TU Dresden, Germany \\ sertkaya@tcs.inf.tu-dresden.de
}

\begin{abstract}
Pseudo-intents play a key rôle in Formal Concept Analysis. They are the premises of the implications in the Duquenne-Guigues Base, which is a minimum cardinality base for the set of implications that hold in a formal context. It has been shown that checking whether a set is a pseudo-intent is in conP. However, it is still open whether this problem is conP-hard, or it is solvable in polynomial time. In the current work we prove a first lower bound for this problem by showing that it is at least as hard as TRANSVERSAL HYPERGRAPH, which is the problem of identifying the minimal transversals of a given hypergraph. This is a prominent open problem in hypergraph theory that is conjectured to form a complexity class properly contained between P and coNP. Our result explains why the attempts to find a polynomial algorithm for recognizing pseudo-intents have failed until now. We also formulate a decision problem, namely FIRST PSEUDO-INTENT, and show that if this problem is not polynomial, then, unless $\mathrm{P}=\mathrm{NP}$, pseudo-intents cannot be enumerated with polynomial delay in a specified lexicographic order.
\end{abstract}

\section{Introduction}

Pseudo-intents play a key rôle in Formal Concept Analysis (FCA) [7]. They form the premises of the Duquenne-Guigues Base [9], which is a minimum cardinality base for the set of implications that hold in a formal context. Computational problems related to pseudo-intents have been of major interest to the FCA community since their introduction. Among these problems, the most central one, namely recognizing pseudo-intents, which is the problem of checking whether a given set is a pseudo-intent of a given formal context, has been shown to be in coNP 15[16. However, so far neither a polynomial time algorithm that solves this problem, nor a proof that this problem is intractable has been found.

In another field of discrete mathematics, namely hypergraph theory [1], problems that show a similar computational behaviour exist as well. The problem known as TRANSVERSAL HYPERGRAPH [3, which is the problem of checking whether the edges of a given hypergraph are precisely the minimal transversals of another given hypergraph, is one such problem. Like the problem of recognizing pseudo-intents, this problem is also known to be in coNP, however whether it

^ Supported by the German Research Foundation (DFG) under grant BA 1122/12-1. 
is conP-hard or it is solvable in polynomial time has now been open for more than 20 years. Moreover, many other problems from various fields of computer science have been shown to be computationally equivalent to this problem. Some of these problems are: from relational databases the problem FD-RELATION EQUIVALENCE, which is checking whether a given set of functional dependencies that is in Boyce-Codd Normal Form is a cover of a given relation instance [3], the problem ADDITIONAL KEY for relation instances, which is the problem of checking whether an additional key exists for a given relation instance and a set of minimal keys thereof 3], and from logic the problem MONOTONE DUAL, which is checking whether two monotone Boolean functions given in CNF are mutually dual [4]. Related problems from artificial intelligence can be found in [13, problems from data mining can be found in 10, and a comprehensive survey on these problems can be found in [4. In a landmark paper Fredman and Khachiyan proved in [5] that TRANSVERSAL HYPERGRAPH can be solved in $n^{o(\log n)}$ time, which implies that this problem is most likely not coNP-hard. It is conjectured that this problem, together with the computationally equivalent problems mentioned above, forms a class properly contained between $\mathrm{P}$ and coNP.

The present paper is the first step of an ongoing work investigating whether the problem of recognizing pseudo-intents is computationally equivalent to the abovementioned problems. We show that it is at least as hard as TRANSVERSAL HYPERGRAPH. However, whether it is TRANSVERSAL HYPERGRAPH-complete remains open. Our result explains why the attempts in the FCA community to find a polynomial time algorithm for this problem have failed until now. We also formulate a decision problem, namely FIRST PSEUDO-INTENT, and show that if this problem is not solvable in polynomial time, then, unless $\mathrm{P}=\mathrm{NP}$, pseudointents cannot be enumerated with polynomial delay in a specified lexicographic order.

\section{Preliminaries}

\subsection{Formal Concept Analysis}

Formal Concept Analysis (FCA) 7] is a field of mathematics that is based on a lattice-theoretic formalization of the notions of a concept and a conceptual hierarchy. It facilitates the use of mathematical reasoning for conceptual data analysis and knowledge processing.

In FCA, one represents data in a formal context, which in its simplest form is a way of specifying which attributes are satisfied by which objects. A formal context is usually denoted by $\mathbb{K}=(G, M, I)$ where $G$ is the set of objects, $M$ is the set of attributes, and $I$ is the incidence relation between the objects and the attributes. A formal context is usually visualized as a cross table, where the rows represent the objects, and the columns represent the attributes of the context. A cross in column $m$ of row $g$ means that the object $g$ has the attribute $m$, and the absence of a cross means that $g$ does not have the attribute $m$. For a set of objects $A \subseteq G$, the derivation operator applied to $A$, which is denoted with $A^{\prime}$, defines the set of attributes that are satisfied by all objects in $A$. Similarly, 
for a set of attributes $B \subseteq M$, the derivation operator applied to $B$, which is denoted with $B^{\prime}$, defines the set of objects that satisfy all attributes in $B$. Double application of the derivation operator yields the closure operator $(\cdot)^{\prime \prime}$. The subsets of $M$ closed under $(\cdot)^{\prime \prime}$ are called the concept intents of $\mathbb{K}$, and when ordered w.r.t. inverse set inclusion, they yield a complete lattice called the concept lattice of $\mathbb{K}$. Given a formal context $\mathbb{K}=(G, M, I)$ and an implication $P \rightarrow Q$, where $P, Q \subseteq M$, we say that $P \rightarrow Q$ holds in $\mathbb{K}$ if the objects that have the attributes in $P$ also have the attributes in $Q$, i.e., $P^{\prime} \subseteq Q^{\prime}$. We denote the implicational theory of $\mathbb{K}$, i.e, the set of all implications that hold in $\mathbb{K}$, with $\operatorname{Imp}(\mathbb{K})$.

The implicational theory of a formal context $\mathbb{K}$ can be large. Thus, one is interested in small implicational bases generating $\operatorname{Imp}(\mathbb{K})$. In 9 a canonical implicational base, which is called the Duquenne-Guigues Base, of a given formal context has been characterized, and it has been shown that there cannot be another base with fewer implications. The premises of the implications in a Duquenne-Guigues Base are called the pseudo-intents of the underlying formal context. A set $P \subseteq M$ is called a pseudo-intent if $P \neq P^{\prime \prime}$ and $Q^{\prime \prime} \subsetneq P$ holds for every pseudo-intent $Q \subsetneq P$. Equivalently, a set $P \subseteq M$ is called a pseudo-intent if $P \neq P^{\prime \prime}$, it is a quasi-intent, and for every quasi-intent $Q \subsetneq P, Q^{\prime \prime} \subsetneq P$ holds, where a quasi-intent is defined as a set $Q \subseteq M$ that satisfies $R^{\prime \prime} \subseteq Q$ or $R^{\prime \prime}=Q^{\prime \prime}$ for any $R \subseteq Q$.

\subsection{Hypergraphs}

Hypergraph theory [1] is a field of discrete mathematics with many important applications in both theoretical and applied computer science.

A hypergraph $\mathcal{H}=(V, \mathcal{E})$ is a pair consisting of a set of vertices $V=\left\{v_{i} \mid\right.$ $1 \leq i \leq n\}$, and a set of (hyper)edges $\mathcal{E}=\left\{E_{j} \mid 1 \leq j \leq m\right\}$ where $E_{j} \subseteq V$. A hypergraph is called simple if none of its edges contains another edge, that is, $\forall E, F \in \mathcal{E} . E \subseteq F \Rightarrow E=F$. A set of vertices $W \subseteq V$ is called a transversal of $\mathcal{H}$ if it intersects every edge of $\mathcal{H}$, i.e., $\forall E \in \mathcal{E}$. $E \cap W \neq \emptyset$. A transversal is called minimal if no proper subset of it is a transversal. The set of all minimal transversals of $\mathcal{H}$ constitute another hypergraph on $V$ called the transversal hypergraph of $\mathcal{H}$, which is denoted by $\operatorname{Tr}(\mathcal{H})$. Generating $\operatorname{Tr}(\mathcal{H})$ is an important problem which has applications in many fields of computer science. The well known decision problem associated to this computation problem is defined as follows:

\section{Problem: TRANSVERSAL HYPERGRAPH (TRANS-HYP)}

Input: Two hypergraphs $\mathcal{H}=\left(V, \mathcal{E}_{\mathcal{H}}\right)$ and $\mathcal{G}=\left(V, \mathcal{E}_{\mathcal{G}}\right)$.

Question: Is $\mathcal{G}$ the transversal hypergraph of $\mathcal{H}$, i.e., does $\operatorname{Tr}(\mathcal{H})=\mathcal{G}$ hold?

We say that a decision problem $\Pi$ is TRANS-HYP-hard if TRANS-HYP can be reduced to $\Pi$ by a standard polynomial transformation. We say that $\Pi$ is TRANSHYP-complete if it is TRANS-HYP-hard and $\Pi$ can be reduced to TRANS-HYP by a polynomial transformation. 


\section{Related Work and Previous Results}

Pseudo-intents and computational problems related to them has attracted great attention among the researchers in the FCA community since their introduction. It is well known that for a formal context $\mathbb{K}=(G, M, I)$ the number of pseudo-intents can be exponential in $|M|$. This is for instance the case when object intents are precisely all possible subsets of $M$ with cardinality $|M| / 2$. However, in this case $|G|$ as well as $|I|$ are also exponential in $|M|$, thus the number of pseudo-intents is polynomial in $|I|$. In 14 Kuznetsov has given an example of a context where the number of pseudo-intents is exponential in the size of the incidence relation $|I|$. Moreover, he has shown that determining the number of pseudo-intents of a formal context is a \#P-hard problem. Given the fact that the number of pseudo-intents can be exponential in the size of the input context, it is clearly not possible to enumerate all pseudointents in time polynomial time in the size of this formal context. In complexity theory, for analyzing the performance of enumeration algorithms where the number of solutions can be exponential in the size of the input, one considers other measures. One such measure is to take into account not only the size of the input, but also the size of the output when defining a notion of performance. An algorithm is said to run in output polynomial time (or polynomial total time) [12] if it outputs all solutions in time polynomial in the size of the input and the output. One advantage of an output polynomial algorithm is that it runs in polynomial time (in the size of the input) when there are only polynomially many solutions.

For enumerating pseudo-intents, currently no output polynomial algorithm is known. The most well known algorithm next closure [6] by Ganter, as a by-product, enumerates the concept intents as well. That is, its running time depends not only on the number of pseudo-intents but also on the number of concept intents. Since the number of concept intents can be exponential in the number of pseudo-intents, this algorithm in general does not run in output polynomial time. Similarly, the attribute-incremental algorithm 17. by Duquenne and Obiedkov has also time complexity depending on both the number of pseudointents and the number of concept intents. Recently in 18] we have shown that enumerating pseudo-intents is at least as hard as computing the minimal transversals of a given hypergraph. There we have also identifed a class of formal contexts for which these two problems are computationally equivalent. In [11], for the special case where the concept lattice is meet-semidistibutive, Janssen and Nourine have shown that there are at most polynomially many pseudo-intents, and they can be enumerated in polynomial time. For the special case where the concept lattice is modular, Wild has shown in 19 that an optimal base, i.e., a base that not only contains the minimum number of implications, but also contains the minimum number of attributes, can be computed in polynomial time. In 2] Duquenne has shown that for locally distributive lattices a minimum cardinality base can be computed in polynomial time. 


\section{Complexity of Recognizing Pseudo-intents}

Apart from enumerating and counting pseudo-intents, recognizing them is another important computatinal problem. Kuznetsov and Obiedkov has shown in [15]16, that this problem is in conP. However, neither a polynomial time algorithm, nor a proof of conP-hardness has been found so far. In the following we prove a first lower bound for this problem.

First we need to introduce some more notions from hypergraphs. A hypergraph $\mathcal{H}=(V, \mathcal{E})$ is called saturated $[3$ if every subset of $V$ is contained in at least one of the edges of $\mathcal{H}$, or it contains at least one edge of $\mathcal{H}$, i.e., for every $W \subseteq V, W \subseteq E$ holds, or $E \subseteq W$ holds for some $E \in \mathcal{E}$. It has been shown in 3 . that checking whether a hypergraph is saturated is conP-complete. There, a special case of the problem where the given hypergraph is restricted to be simple, has also been considered. It is the following problem:

\section{Problem: SIMPLE hyPERGRAPH SATURATION (SIMPLE-H-SAT)}

Input: A simple hypergraph $\mathcal{H}=(V, \mathcal{E})$, i.e., $\forall E, F \in \mathcal{E} . E \subseteq F \Rightarrow E=F$.

Question: Is $\mathcal{H}$ saturated, i.e., is it true that for every $W \subseteq V, W \subseteq E$ holds or $E \subseteq W$ holds for some $E \in \mathcal{E}$ ?

It is not difficult to see that this problem is in coNP. However, like the problem of recognizing pseudo-intents, neither a polynomial time algorithm that solves this problem, nor a proof that it is coNP-hard has been found so far. It has been shown in [3] that this problem is under polynomial transformations computationally equivalent to TRANS-HYP. In the following we show that recognizing pseudointents is at least as hard as this problem. We start with a formal definition of our problem:

\section{Problem: PSEUdO-INTENT (PSI)}

Input: A formal context $\mathbb{K}=(G, M, I)$, and a set $P \subseteq M$.

Question: Is $P$ a pseudo-intent of $\mathbb{K}$ ?

Now we show that PSI is SIMPLE-H-SAT-hard.

Theorem 1. PSI is SIMPLE-H-SAT-hard.

Proof. Let an instance of SIMPLE-H-SAT be given with the simple hypergraph $\mathcal{H}=(V, \mathcal{E})$, where $V=\left\{v_{1}, \ldots, v_{n}\right\}$ and $\mathcal{E}=\left\{E_{1}, \ldots, E_{m}\right\}$. From $\mathcal{H}$ we construct an instance of PSI, i.e., a formal context $\mathbb{K}_{\mathcal{H}}=(G, M, I)$ and a set $P \subseteq M$, as follows: As attributes of $\mathbb{K}_{\mathcal{H}}$, we take the vertices of $\mathcal{H}$ and two new attributes $a$ and $b$ that do not already occur in $V$, that is, $M=V \cup\{a, b\}$. For every $i$, where $1 \leq i \leq m$, we construct an object $g_{i}$ whose intent is $E_{i} \cup\{a\}$. In addition, for each $i$ we construct the following objects: Consider edge $E_{i}$. For every $F \subsetneq E_{i}$ such that $|F|=\left|E_{i}\right|-1$, we create an object with the intent $F$. $E_{i}$ has $\left|E_{i}\right|$-many such subsets. We name these objects as $g_{i 1}, \ldots, g_{i\left|E_{i}\right|}$. In total $\mathbb{K}_{H}$ contains $\sum_{i=1}^{m}\left|E_{i}\right|+m$ objects. Figure 1 demonstrates the context $\mathbb{K}_{\mathcal{H}}$. Finally we create a subset of $M$ just by defining $P=V \cup\{a\}$. It is easy to see that both $\mathbb{K}_{\mathcal{H}}$ and $P$ can be constructed in polynomial time. We know that $\mathcal{H}$ is simple, 


\begin{tabular}{|c|c|c|}
\hline & $v_{1} \quad \ldots$ & $v_{n}|a|$ \\
\hline$g_{1}$ & $E_{1}$ & $\mathrm{x}$ \\
\hline : & : & : \\
\hline$g_{m}$ & $E_{m}$ & $\mathrm{x}$ \\
\hline$g_{11}$ & $F_{11}$ & \\
\hline : & : & \\
\hline$g_{1\left|E_{1}\right|}$ & $F_{1\left|E_{1}\right|}$ & \\
\hline$\vdots$ & $\vdots$ & \\
\hline$g_{m 1}$ & $F_{m 1}$ & \\
\hline$\vdots$ & & \\
\hline$g_{m\left|E_{m}\right|}$ & $F_{m\left|E_{m}\right|}$ & \\
\hline
\end{tabular}

Fig. 1. Formal context $\mathbb{K}_{\mathcal{H}}$ constructed from simple hypergraph $\mathcal{H}$

that is none of its edges is contained in another edge. Then $\mathbb{K}_{\mathcal{H}}$ has the following property:

(*) Each $E_{i}$ is contained in only one object intent, namely in $E_{i} \cup\{a\}$, so $E_{i}^{\prime \prime}=$ $E_{i} \cup\{a\}$. That is, $E_{i}$ are not closed. Moreover, strict subsets of $E_{i}$ are closed. In order to see this, consider a fixed $E_{i}$. $\mathbb{K}_{\mathcal{H}}$ contains the objects $g_{i 1}, \ldots, g_{i\left|E_{i}\right|}$ whose intents are all strict subsets of $E_{i}$ with cardinality $\left|E_{i}\right|-1$. Every strict subset of $E_{i}$ can be written as the intersection of such object intents, i.e, every strict subset of $E_{i}$ is closed. This means that the edges of $\mathcal{H}$ are pseudo-intents of $\mathbb{K}_{\mathcal{H}}$.

Now we claim that $P$ is a pseudo-intent of $\mathbb{K}_{\mathcal{H}}$ if and only if $\mathcal{H}$ is saturated. We are going to give a proof of the contrapositive of this claim, i.e., $\mathcal{H}$ is not saturated if and only if $P$ is not a pseudo-intent of $\mathbb{K}_{\mathcal{H}}$.

$(\Rightarrow)$ If $\mathcal{H}$ is not saturated, then there exists a $W \subseteq V$ such that $W \nsubseteq E_{i}$ and $E_{i} \not \subseteq W$ for every $1 \leq i \leq m$. Then $W^{\prime}=\emptyset$, and $W^{\prime \prime}=M$ because $W$ is not contained in any object intent. Assume without loss of generality that $W$ is minimal with respect to set inclusion, that is for every $X \subsetneq W, X \subseteq E_{i}$ holds for some $i$. We know that $E_{i} \nsubseteq W$ holds for every $i$. Then every $X \subsetneq W$ is strictly contained in some $E_{i}$, that is $X \subsetneq E_{i}$ for some $i$. By property (*) above we know that strict subsets of $E_{i}$ are closed, thus $X$ is closed. That is $W$ is not closed, but its all strict subsets are closed, i.e., $W$ is a pseudo-intent of $\mathbb{K}_{\mathcal{H}}$. Since $W \subsetneq P$ and $W^{\prime \prime}$ is not strictly contained in $P, P$ is not a pseudo-intent of $\mathbb{K}_{\mathcal{H}}$. Thus we have shown that if $\mathcal{H}$ is not saturated, then $P$ is not a pseudo-intent of $\mathbb{K}_{\mathcal{H}}$.

$(\Leftarrow)$ If $P$ is not a pseudo-intent of $\mathbb{K}_{\mathcal{H}}$, then $\mathbb{K}_{\mathcal{H}}$ has a pseudo-intent $W \subsetneq P$ such that $W^{\prime \prime}$ is not strictly contained in $P$ (this is because $P$ is not closed). It cannot be the case that $W^{\prime \prime}=P$ since $\mathbb{K}_{\mathcal{H}}$ does not contain any object whose intent is $P$ or a superset of $P$. This means that $W^{\prime \prime}=M$, i.e., $W^{\prime}=\emptyset$, that is $W$ is not contained in any object intent. Assume $a \in W$. We know that $W$ is not contained in any object intent. This implies that $W \backslash\{a\}$ is not contained in any object intent either, i.e., $(W \backslash\{a\})^{\prime \prime}=M$. Note that $(W \backslash\{a\})$ is a quasi-intent, because every $X \subseteq(W \backslash\{a\})$ satisfies $X^{\prime \prime} \subseteq(W \backslash\{a\})$, or $X^{\prime \prime}=(W \backslash\{a\})^{\prime \prime}=M$. 
But this contradicts the fact that $W$ is a pseudo-intent since a pseudo-intent is minimal among the quasi-intents that generate the same closure. Thus, $a \notin W$, i.e., $W \subseteq V$. Due to property (*) each edge $E_{i}$ of $\mathcal{H}$ is a pseudo-intent of $\mathbb{K}_{\mathcal{H}}$ and its closure contains the attribute $a$. Since a pseudo-intent contains the closure of all strictly smaller pseudo-intents and $a \notin W$, for every $1 \leq i \leq m, E_{i}$ is not a strict subset of $W$. In addition $W$ is different from every $E_{i}$ since $W^{\prime \prime} \neq E_{i}^{\prime \prime}$ for every $i$. That is, $E_{i} \nsubseteq W$ for every $1 \leq i \leq m$. Moreover, since $W$ is a pseudointent and its closure $W^{\prime \prime}=M$ is not contained in any $E_{i}, W$ is not contained in any $E_{i}$, i.e., for every $1 \leq i \leq m, W \nsubseteq E_{i}$. Thus we have shown that if $P$ is not a pseudo-intent of $\mathbb{K}_{\mathcal{H}}$, then there is a $W \subseteq V$ such that $W \nsubseteq E_{i}$ and $E_{i} \nsubseteq \mathbb{W}$ for every $1 \leq i \leq m$, i.e., $\mathcal{H}$ is not saturated. This completes the proof of our claim.

The following is an immediate consequence of Theorem 1 above and Theorem 4.12 in 3$]$ :

\section{Corollary 1. PSI is TRANS-HYP-hard.}

Corollary 1 explains why attempts to find a polynomial time algorithm for solving PSI have failed until now. Because if such an algorithm exists, then this algorithm can also decide TRANS-HYP in polynomial time.

\section{Enumerability with Polynomial Delay}

One other notion for analyzing the performance of enumeration algorithms is polynomial delay. An algorithm is said to run with polynomial delay [12] if the time until the first solution is generated, and thereafter the time between any two consecutive solutions is bounded by a polynomial in the size of the input. Currently we do not know whether pseudo-intents can be enumerated with polynomial delay in a specified order. Assuming that the elements of $M$ are linearly ordered, we say that a set $P \subseteq M$ is lexicographically smaller than $Q \neq P$ if the smallest element that distinguishes $P$ and $Q$ belongs to $Q$. The following decision problem is of crucical importance for the above question:

\section{Problem: FIRST PSEUDO-INTENT (FIRST-PSI)}

Input: A formal context $\mathbb{K}=(G, M, I)$, a pseudo-intent $P \subseteq M$ of $\mathbb{K}$, and a linear order on $M$.

Question: Is $P$ lexicographically the first pseudo-intent of $\mathbb{K}$ ?

Because if FIRST-PSI cannot be decided in polynomial time, then pseudo-intents cannot be enumerated with polynomial delay in a specified lexicographic order.

Proposition 1. If FIRST-PSI is not in $\mathrm{P}$, then unless $\mathrm{P}=\mathrm{NP}$, pseudo-intents cannot be enumerated in lexicographic order with polynomial delay.

Proof. It is not difficult to see this. Assume we have an algorithm that enumerates the pseudo-intents of a given formal context with polynomial delay. This 
means that given a formal context $\mathbb{K}=(G, M, I)$ and a $P \subseteq M$, it generates the lexicographically next pseudo-intent coming after $P$ in polynomial time. If we run this algorithm with the input $\mathbb{K}$ and $P=\emptyset$, which is the lexicographically smallest subset of $M$, then in polynomial time it generates the lexicographically first pseudo-intent, thus solves FIRST-PSI in polynomial time.

Of course if PSI turns out to be conP-hard, then unless $\mathrm{P}=\mathrm{NP}$, pseudo-intents cannot be enumerated with polynomial delay since the lexicographically first pseudo-intent cannot be generated in polynomial time. However, even if PSI turns out to be polynomial it can still be the case that FIRST-PSI is intractable. A similar case about maximal independent sets has been investigated in [12]. Although recognizing a maximal independet set is polynomial, there it has been shown that deciding whether a given set is the lexicographically last maximal independent set, is conP-hard. Thus maximal independent sets cannot be enumerated in reverse lexicographic order with polynomial delay.

\section{Concluding Remarks and Future Work}

We have shown that recognizing pseudo-intents is at least as hard as recognizing the transversal hypergraph, which is a prominent open problem. This can be taken as a weak evidence that recognizing pseudo-intents is unlikely to be polynomial time solvable. As future work we are going to work further on investigating whether these problems are computationally equivalent, i.e., whether PSI is TRANS-HYP-complete. We are also going to work on solvability of PSI with limited non-determinism [8], and determining the complexity of FIRST-PSI.

\section{References}

1. Berge, C.: Hypergraphs. Elsevier Science Publishers B.V, North Holland (1989)

2. Duquenne, V.: The core of finite lattices. Discrete Mathematics 88, 133-147 (1991)

3. Eiter, T., Gottlob, G.: Identifying the minimal transversals of a hypergraph and related problems. SIAM Journal on Computing 24(6), 1278-1304 (1995)

4. Eiter, T., Gottlob, G.: Hypergraph transversal computation and related problems in logic and AI. In: Flesca, S., Greco, S., Leone, N., Ianni, G. (eds.) JELIA 2002. LNCS, vol. 2424, pp. 549-564. Springer, Heidelberg (2002)

5. Fredman, M.L., Khachiyan, L.: On the complexity of dualization of monotone disjunctive normal forms. Journal of Algorithms 21(3), 618-628 (1996)

6. Ganter, B.: Two basic algorithms in concept analysis. Technical Report PreprintNr. 831, Technische Hochschule Darmstadt, Darmstadt, Germany (1984)

7. Ganter, B., Wille, R.: Formal Concept Analysis: Mathematical Foundations. Springer, Berlin (1999)

8. Goldsmith, J., Levy, M., Mundhenk, M.: Limited nondeterminism. SIGACT 27(2), 20-29 (1978)

9. Guigues, J.-L., Duquenne, V.: Familles minimales d'implications informatives resultant d'un tableau de données binaries. Mathématiques, Informatique et Sciences Humaines 95, 5-18 (1986) 
10. Gunopulos, D., Khardon, R., Mannila, H., Toivonen, H.: Data mining, hypergraph transversals, and machine learning. In: Proceedings of the Sixteenth Symposium on Principles of Database Systems (PODS 1997), pp. 209-216 (1997)

11. Janssen, P., Nourine, L.: Minimum implicational basis for meet-semidistributive lattices. Information Processing Letters 99(5), 199-202 (2006)

12. Johnson, D.S., Papadimitriou, C.H., Yannakakis, M.: On generating all maximal independent sets. Information Processing Letters 27(3), 119-123 (1988)

13. Kavvadias, D.J., Papadimitriou, C.H., Sideri, M.: On horn envelopes and hypergraph transversals. In: Ng, K.W., Balasubramanian, N.V., Raghavan, P., Chin, F.Y.L. (eds.) ISAAC 1993. LNCS, vol. 762, pp. 399-405. Springer, Heidelberg (1993)

14. Kuznetsov, S.O.: On the intractability of computing the Duquenne-Guigues Base. Journal of Universal Computer Science 10(8), 927-933 (2004)

15. Kuznetsov, S.O., Obiedkov, S.A.: Counting pseudo-intents and \#P-completeness. In: Missaoui, R., Schmidt, J. (eds.) Formal Concept Analysis. LNCS, vol. 3874, pp. 306-308. Springer, Heidelberg (2006)

16. Kuznetsov, S.O., Obiedkov, S.A.: Some decision and counting problems of the Duquenne-Guigues basis of implications. Discrete Applied Mathematics 156(11), 1994-2003 (2008)

17. Obiedkov, S.A., Duquenne, V.: Attribute-incremental construction of the canonical implication basis. Annals of Mathematics and Artificial Intelligence 49(1-4), 77-99 (2007)

18. Sertkaya, B.: Some computational problems related to pseudo-intents. In: Ferré, S., Rudolph, S. (eds.) Proceedings of the 7th International Conference on Formal Concept Analysis (ICFCA 2009). LNCS (LNAI), vol. 5548, pp. 130-145. Springer, Heidelberg (2009)

19. Wild, M.: Optimal implicational bases for finite modular lattices. Quaestiones Mathematicae 23, 153-161 (2000) 